27th EPS Conference on Contr. Fusion and Plasma Phys. Budapest, 12-16 June 2000 ECA Vol. 24B (2000) 988-991

\title{
Fast Ion Losses in the W7-AS Stellarator
}

\author{
A. Werner, A.Weller, D.S. Darrow* and the W7-AS Team \\ Max Planck Institut für Plasmaphysik, EURATOM Association, Boltzmannstr. 2, D-85748 \\ Garching, Germany \\ * Princeton Plasma Physics Laboratory, Princeton, New Jersey 08543-0451, USA
}

\section{INTRODUCTION}

Good $\alpha$-particle confinement is essential for future nuclear fusion reactors to retain a magnetically confined plasma in the burning state. Thus, the study of fast ion confinement in the present nuclear fusion experiments receives increasing attention. In particular, stellarators with non-axisymmetric field topology suffer from trapped ion losses due to local poloidal and toroidal magnetic mirrors.

The $\alpha$-particle confinement of tokamaks was studied extensively in DT-experiments of TFTR [1][2], in stellarators losses of fast ions, generated by auxiliary heating, were investigated in the Compact Helical System (CHS) [3]. The modular stellarator W7-AS has a partially drift-optimized magnetic field topology giving rise to a particular interest in the fast ion confinement studies. In general, these studies are related to the following issues: (I) If fast ions are not confined long enough to transfer their energy to the bulk plasma, $\alpha$-particle heating is deteriorated and the efficiencies of NBI (section 3 ) and ICRH (section 4) are reduced; (II) If fast ions are lost to the wall, excessive heat loads and additional impurity sources are generated (section 3), leading to radiation power losses and endangering stationary plasma operation in plasmas with good impurity confinement. (III) MHD perturbations may induce fast ion losses even in well confining stationary fields (section 5). However, this effect could also provide a possibility of helium ash removal from fusion plasmas.

At W7-AS, fast ion losses have been measured using a probe based on the concept of the TFTR $\alpha$-particle detectors [4]. The probe design, similar to the CHS probe [5], was extended to simultaneously measure both co- and counter-going losses simultaneously.

\section{FAST ION LOSS DIAGNOSTIC}

The fast ion loss probe of W7-AS is depicted schematically in Fig. 1. The main part consists of an aperture system (a pinhole followed by a slit) and a scintillator plate behind. Ions, gyrating through the apertures, are dispersed in gyroradius $\left(\rho=\sqrt{\left(2 M E_{\text {ion }}\right) / q B}\right.$, $E_{i o n}$ being the full kinetic energy, for convenience) and in pitch angle $\left(\chi=\cos ^{-1}\left(v_{\|} / v\right)\right)$. Thus, the scintillator light pattern on the plate gives the fast ion loss distribution in two velocity components at the detector position. It is viewed through an optical system by a CCD camera (128x128 pixel, $13 \mathrm{~Hz}$ ) and additionally by 15 photomultipliers (4 or 100 $\mathrm{kHz}$ ) enabling high spatial and temporal resolution.

In principle, absolute loss rates could be determined from the yield of scintillator light being a function of particle energy. However, this requires precise knowledge of the scintillator efficiency. A much more simple access to absolute rates can be obtained by using the probe as a Faraday cup measuring directly the current of striking ions. Thus, the glass substrate of the plate was coated with a Cr-layer before the scintillator powder (P11) was deposited in a sedimentation process.

The new feature of the W7-AS probe is the capability to measure both co- and countergoing losses simultaneously. This was achieved by integrating two such systems into one casing. The scintillator material as well as the Cr-layer was separated into two 
27th EPS CCFPF 2000; A. Werner et al.: Fast Ion Losses in the W7-AS Stellarator

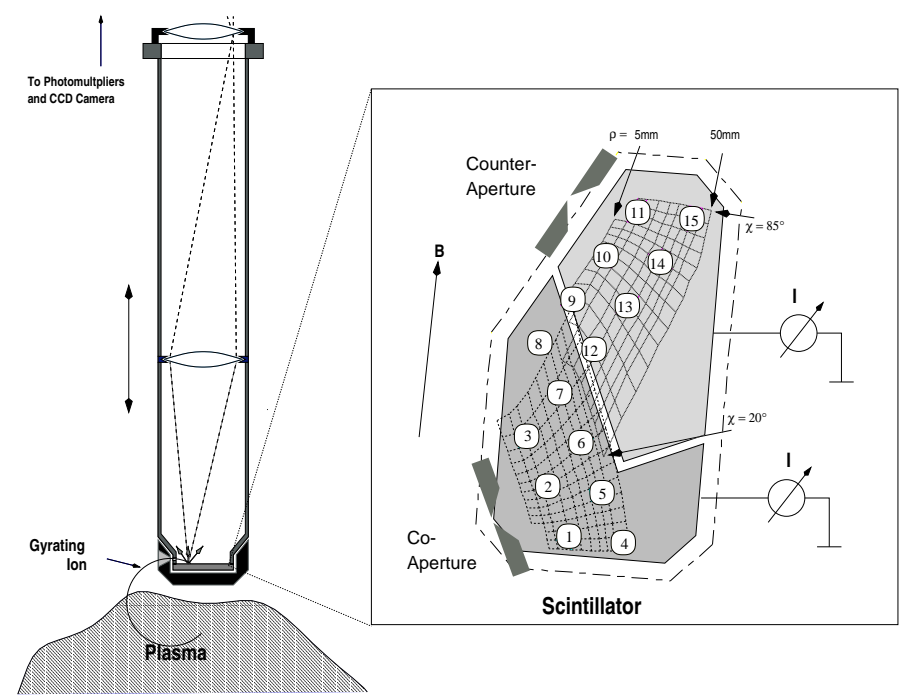

Fig. 1: Probe shaft and scintillator layout of the fast ion loss detector. Calculated maps in $(\rho, \chi)$ and the viewing points of the photomultipliers are overlaid. A wide range of the detector acceptance, $\rho=5 \ldots 50 \mathrm{~mm} \Rightarrow$ $E_{\text {ion }} \approx 5 \mathrm{keV} \ldots 500 \mathrm{keV}$ and $\chi=$ $15^{\circ} \ldots 85^{\circ}$, is achieved by minimizing ion orbit scrape-off at the shielding graphite with $M C$ calculations.

domains and two staggered aperture systems were placed in front of it, so that both loss distributions do not significantly overlap.

The probe location at W7-AS was chosen appropriately to the field topology. In order to detect trapped ion losses, the probe has to be placed in local minimum of $B_{\text {mod }}$. Passing ion losses can be detected at any toroidal angle, because such losses are caused by the displacement of the drift orbits to flux surface. Since this displacement is oriented horizontally and the drift motion is upwards in the normal field direction of W7-AS, the probe was placed on top of the machine at $\varphi=27.5^{\circ}\left(\varphi=0\right.$ triangular plane, $\varphi=36^{\circ}$ elliptical plane; see [6] for W7-AS geometry). The probe position in vertical direction is variable allowing a positioning close to the plasma edge.

\section{NEUTRAL BEAM INJECTION}

Fast beam ion losses have been observed during perpendicular and tangential neutral beam injection. The perpendicular beam is generated by the vertically injecting diagnostic injector, which is located stellarator symmetric to the probe in the same field period ( W7-AS has 5 field periods [6]). Beam ions, born below the equatorial midplane, are
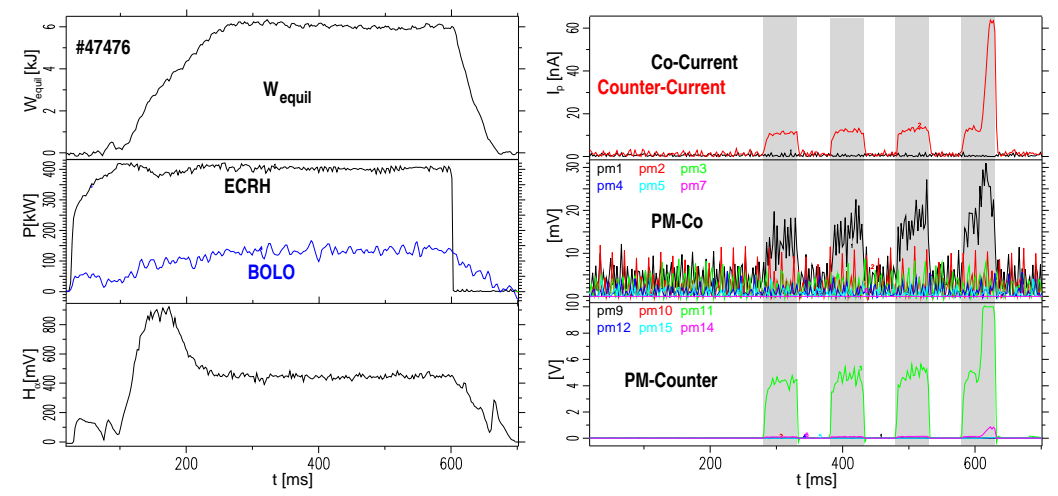

Fig. 2: Probe signals during diagnostic beam injection (shaded intervals) into an ECRH discharge. The co-signals are strongly suppressed by enhanced field ripple at $\varphi=36^{\circ}$. Trapped ion losses occur exclusively during beam injection.

trapped in a local mirror and drift towards the magnetic axis, where the field ripple is low. There, moving toroidally to the adjacent half period, they become trapped again and bounce upwards. This provides a perfect test facility for the loss probe. Corresponding probe signals are shown in Fig. 2.

An interesting feature occurs for tangential beam injecting. Although the mean pitch angle of the beam ions $\left\langle\chi>\approx 30^{\circ}\right.$, trapped ion losses are often observed beside the 
27th EPS CCFPF 2000; A. Werner et al.: Fast Ion Losses in the W7-AS Stellarator

passing ones.

The fact, that trapped ion losses can only occur if beam ions were scattered in pitch angle, gives evidence that the slowing down process has to be taken into account for an explanation of the observation. As a consequence, fast ion losses in W7AS depend on all plasma parameters affecting the slowing down of fast ions, like electron and ion temperature, density and the effective charge $Z_{\text {eff }}$. The influence of $Z_{\text {eff }}$ is observed in discharges with combined ECRH and NBI in good confinement regimes of $t=0.34$, where large critical energies in the order of $40 \mathrm{keV}$ were achieved in the slowing down. In such discharges, ion-ion slowing dominates, so that light beam ions, scattered on heavy impurity ions, change the direction rather than the magnitude of momentum. Trapped ion fluxes, slowly increasing in time, are observed whereas the passing ion fluxes remain almost constant. A direct correlation to $Z_{\text {eff }}$ obtained by Thomson-YAG diagnostics is found experimentally.

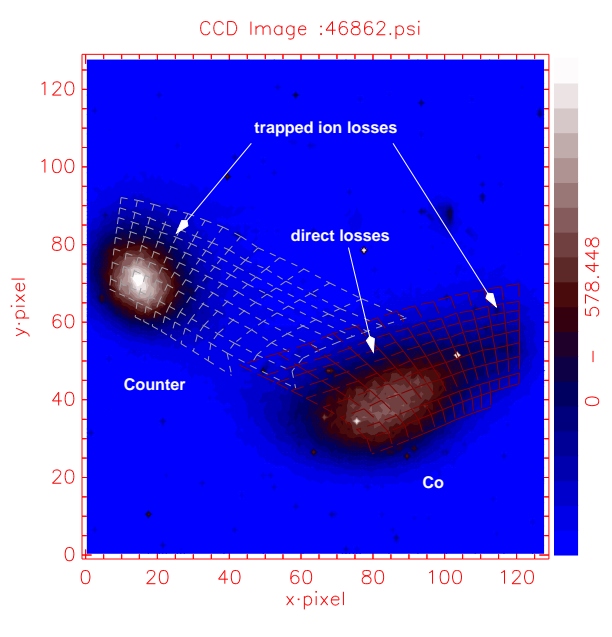

Fig. 3: $C C D$ image recorded during tangential co-NBI. Losses at low pitch angle on the co-side are associated with first orbit losses. Trapped ion losses at large pitch angles are associated with partially slowed down beam ions.

\section{ION CYCLOTRON HEATING}

Ions, heated by ion cyclotron heating, predominantly gain perpendicular momentum. Thus, accelerated ions reside or enter the loss cone of the configuration and could be detected with the loss probe, provided that they are not slowed down before crossing the last closed flux surface.

If no Alfv́en or kinetic instabilities are driven by ICRH, fast ion loss rates can be related directly to the heating efficiency. This is investigated theoretically in [7]. The data shown in Fig. 4 exhibit experimental evidence for heating power losses by fast ions. The applied power is increased stepwise and a significant change in the stored energy is only observed for $P_{\mathrm{RF}}<400 \mathrm{~kW}$. The subsequent power steps lead to an increase of ion loss fluxes but not to an increase of plasma energy. The CCD data, not shown here, indicate losses at $\chi \approx 50^{\circ}$ and low $\rho$ with a broad distribution in $\chi$ for intermediate $P_{\mathrm{RF}}$. At the largest $P_{\mathrm{RF}}$, a clear evolution of an energetic ion tail at $\chi \approx 80^{\circ}$ is observed.
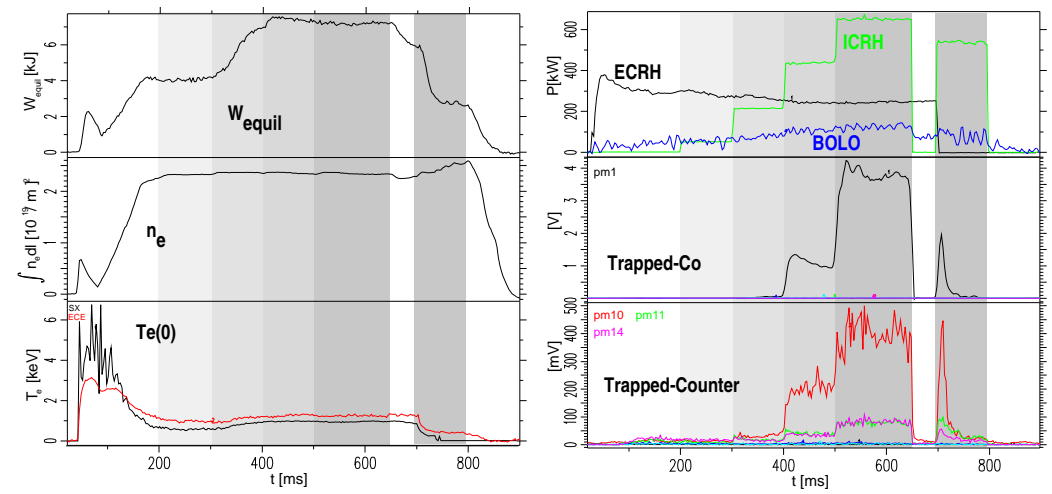

Fig. 4: ICR minority heating in an ECRH discharge (\#47024). The RF power is increased stepwise. Trapped ion losses at large $P_{R F}$ are found.

At the beginning of the last ICRH pulse, ECRH is switched off. The electron temperature drops, the fast ion losses vanish and the ICRH is capable of sustaining the plasma. Provided that no instabilities are driven (at least no Alfvén eigenmodes have been observed by Mirnov diagnostics), it is an clear indication that ions are lost, if they are 
27th EPS CCFPF 2000; A. Werner et al.: Fast Ion Losses in the W7-AS Stellarator

accelerated to large energies at which slowing down is insufficient. Thus, energetic ions generated by ICRH are lost before they transfer energy to the bulk plasma. In largely drift-optimized configurations, like W7-X [8], the performance of ICRH should be better in such cases.

\section{MHD INDUCED LOSSES}

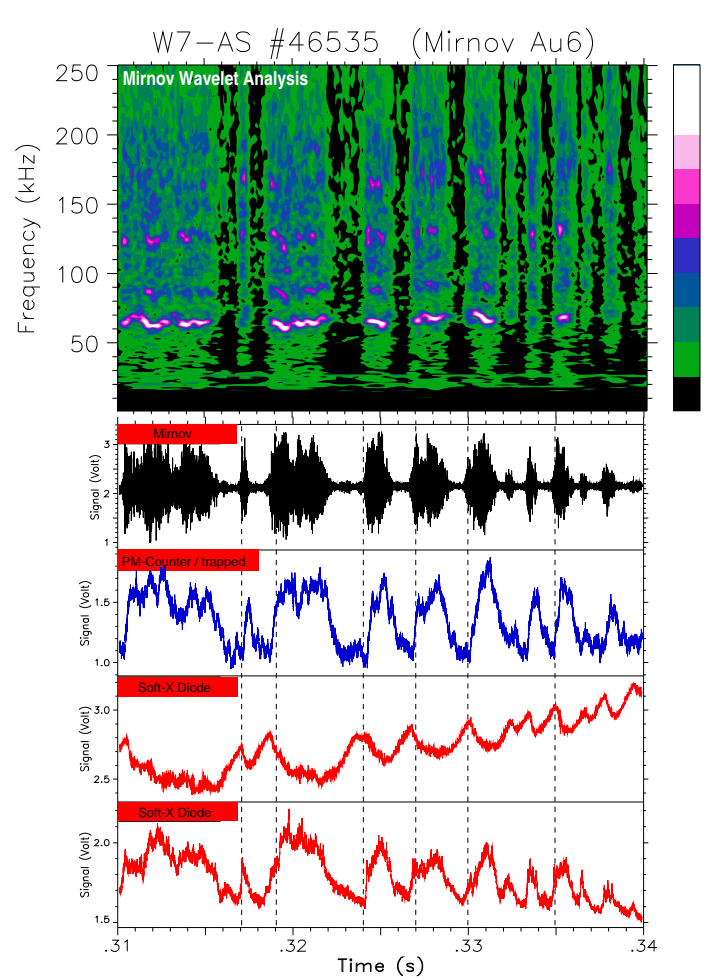

Fig. 5: Mirnov frequency spectrum and signal, trapped ion losses and soft- $X$ signals of discharge \#46535. Enhanced ion losses obviously correlated to MHD activity. less than the enhancement factor up to 30 observed at CHS [10].

\section{References}

[1] Zweben S J et al., Nucl. Fusion 40, 91 (2000)

[2] Heidbrink W W and Sadler G, Nucl. Fusion 34, 535 (1994)

[3] Isobe $\mathrm{M}$ et al., Rev. Sci. Instrum. 70, 827 (1999)

[4] Darrow D S, Rev. Sci. Instrum. 63, 4565 (1995)

[5] Darrow D S et al., Rev. Sci. Instrum. 70, 838 (1999)

[6] Hofmann J V et al., Plasma Phys. Control. Fusion 38, A193 (1996)

[7] Murakami S et al., Nucl. Fusion 39, 1165 (1999)

[8] Grieger G et al., Phys. Fluids B 4, 2081 (1992)

[9] Sigmar D J et al., Phys. Fluids B 4, 1506 (1992)

[10] Isobe $\mathrm{M}$ et al., in Europhys. Conf. Abstr. 23J, 21 (1999) 